Wright State University

CORE Scholar

Mechanical and Materials Engineering Faculty

Publications

Mechanical and Materials Engineering

9-1997

\title{
Optimization of Microstructure Development During Hot Working Using Control Theory
}

James C. Malas III

Wright Patterson Air Force Base

W. Garth Frazier

Wright Patterson Air Force Base

S. Venugopal

Wright Patterson Air Force Base

Enrique A. Medina

Wright Patterson Air Force Base

Steven Madeiros

Wright Patterson Air Force Base

See next page for additional authors

Follow this and additional works at: https://corescholar.libraries.wright.edu/mme

Part of the Materials Science and Engineering Commons, and the Mechanical Engineering Commons

\section{Repository Citation}

Malas, J. C., Frazier, W. G., Venugopal, S., Medina, E. A., Madeiros, S., Srinivasan, R., Irwin, R. D., Mullins, W. M., \& Chaudhary, A. (1997). Optimization of Microstructure Development During Hot Working Using Control Theory. Metallurgical and Materials Transactions A: Physical Metallurgy and Materials Science, 28A (9), 1921-1930.

https://corescholar.libraries.wright.edu/mme/3

This Article is brought to you for free and open access by the Mechanical and Materials Engineering at CORE Scholar. It has been accepted for inclusion in Mechanical and Materials Engineering Faculty Publications by an authorized administrator of CORE Scholar. For more information, please contact library-corescholar@wright.edu. 


\section{Authors}

James C. Malas III, W. Garth Frazier, S. Venugopal, Enrique A. Medina, Steven Madeiros, Raghavan Srinivasan, R. Dennis Irwin, W. M. Mullins, and Anil Chaudhary 


\title{
Optimization of Microstructure Development during Hot Working Using Control Theory
}

\author{
JAMES C. MALAS III, W. GARTH FRAZIER, S. VENUGOPAL, ENRIQUE A. MEDINA, \\ STEVEN MEDEIROS, RAGHAVAN SRINIVASAN, R. DENNIS IRWIN, W. M. MULLINS, \\ AND ANIL CHAUDHARY
}

\begin{abstract}
A new approach for controlling microstructure development during hot working processes is proposed. This approach is based on optimal control theory and involves state-space type models for describing the material behavior and the mechanics of the process. The effect of process control parameters such as strain, strain rate, and temperature on important microstructural features can be systematically formulated and then solved as an optimal control problem. This method has been applied to the optimization of grain size and process parameters such as die geometry and ram velocity during the extrusion of plain carbon steel. Experimental results of this investigation show good agreement with those predicted in the design stage.
\end{abstract}

\section{INTRODUCTION}

THE development of optimal design and control methods for manufacturing processes is needed for effectively reducing part cost, improving part delivery schedules, and producing specified part quality on a repeatable basis. Existing design methods are generally ad hoc and lack adequate capabilities for finding effective process parameters such as deformation rate, die and workpiece temperature, and tooling system configuration. This situation presents major challenges to process engineers who are faced with smaller lot sizes, higher yield requirements, and superior quality standards. For example, the design of near-netshape processes with precisely controlled microstructure and properties poses difficult problems not addressed by traditional techniques. Therefore, it is important to develop new systematic methodologies for process design and control based upon scientific principles, which sufficiently consider the behavior of workpiece material and the mechanics of the manufacturing process.

A new strategy for systematically calculating near optimal control parameters for control of microstructure during hot deformation processes has been developed based on optimal control theory..$^{[1]}$ This approach treats the deforming material as a dynamical system and involves developing state-space models ${ }^{[2]}$ from available material behavior and hot deformation process models. Modeling of dynamical systems in state-space form provides a natural framework for describing the time evolution of the states and outputs of a system under the influence of multiple, time-varying

JAMES C. MALAS III, Research Leader, W. GARTH FRAZIER, ENRIQUE A. MEDINA, STEVEN MEDEIROS, W.M. MULLINS, and ANIL CHAUDHARY, Visiting Scientists, and S. VENUGOPAL, Resident Research Associate, on leave from the Indira Gandhi Centre for Atomic Research, Kalpakkam, India, are with the Materials Process Design Branch, Materials Directorate, Wright Laboratory, Wright Patterson Air Force Base, OH 45433-7995. R. DENNIS IRWIN, Professor, is with the School of Electrical Engineering and Computer Science, Ohio University, Athens, OH 45701. RAGHAVAN SRINIVASAN, Associate Professor, is with the Department of Mechanical and Materials Engineering, Wright State University, Dayton, OH 45435.

Manuscript submitted August 6, 1996. inputs. Also, the wealth of the analytical and computational tools available for analyzing and designing optimal controls for these system models is enormous, thereby facilitating reliable process analysis and design. The successful use of state-space modeling and design techniques for dynamical systems in areas such as electrical, mechanical, chemical, and aerospace systems has been demonstrated. ${ }^{[3,4]}$ The extension of these techniques to material systems, while complex, is quite natural.

Two stages of analysis and optimization form the basis of this strategy for the control system design. In the first stage, the optimal strain, strain rate, and temperature trajectories* for the "safe" processing of the material are cal

*The variation of strain, strain rate, and temperature as a function of time.

culated. These trajectories are obtained from the kinetics of certain dynamic microstructural behaviors (for example, dynamic recrystallization), thermophysical characteristics of the material, and intrinsic hot workability of the material, along with an optimality criterion** chosen by the designer.

**A mathematical expression that provides a quantitative measure of the quality of the design.

If the deforming material follows these optimal trajectories, it will not fail during processing, and the product will possess the desired microstructural features. These trajectories are predominantly governed by the workpiece material and its thermomechanical history rather than by the type of hot deformation process, e.g., forging, rolling, or extrusion. In the second stage, an appropriate process simulation model is used to calculate process control parameters such as ram velocity, die shape, and billet temperature for a particular deformation process, which ensure that the material follows the strain, strain rate, and temperature trajectories calculated in the first stage. Current process models, which are required in stage 2, are capable of analyzing fairly complex material flow operations such as three-dimensional, nonisothermal deformation processes with a sufficiently high degree of accuracy.

This paper is aimed at explaining a new approach for the design and control of microstructure during hot deformation 
processes. The important steps involved in the formulation of the optimal control problem and optimality criteria are briefly discussed, and the validity of this approach is demonstrated with an example of hot steel extrusion. An extrusion process was selected for study for the following reasons: (1) extrusion typically involves large deformation with large variations in strain rate, (2) relatively simple analytical models are available for describing the process, and (3) strain and strain rate trajectories can be effectively controlled via proper design of die geometry. In the present investigation, the optimal die profile, ram velocity, and billet temperature have been obtained to achieve a desired grain size in the extrudate of AISI 1030 steel, using the proposed methodology. Detailed experimental study has been undertaken to validate this new technique.

\section{TWO-STAGE APPROACH TO OPTIMAL CONTROL OF DEFORMATION PROCESSES}

As shown in Figure 1, the design methodology is separated into a microstructure optimization stage (or problem) and a process optimization stage (or problem). Goals of the first stage are to achieve enhanced workability and to obtain prescribed microstructural parameters. In this investigation, an attempt has been made to control microstructural parameters such as final grain size (average) and volume fraction recrystallized during deformation. In the second stage, a primary goal is to achieve the thermomechanical conditions required by the first stage for predetermined regions of the deforming workpiece. In Figure 1, desired microstructural features, such as volume fraction recrystallized and grain size, serve as inputs to the microstructure optimization problem. Trajectories of strain, strain rate, and temperature are the outputs, which depend on the details of the microstructure development model and the criterion used for optimization. Next, these trajectories become the inputs to the process optimization problem. The outputs of the process optimization are the die shape, ram velocity, and billet temperature for the case of extrusion.

The design approach requires three basic components for defining and setting up the optimization problem: (1) a dynamical system model; (2) physical constraints; and (3) an optimality criterion. In metal forming, the system models of interest are material behavior and deformation process models; constraints include the hot workability of the workpiece and the limitations of the forming equipment. Optimality criteria can be chosen to achieve a particular set of final microstructural features, to regulate temperature, and to maximize deformation speeds.

\section{A. Material Behavior and Process Modeling}

Material behavior models that describe the kinetics of primary metallurgical mechanisms such as dynamic recovery, dynamic recrystallization, and grain growth during hot working are required for analysis and optimization of material system dynamics. These mechanisms have been studied extensively for a wide range of metals and alloys. ${ }^{[5-9]}$ The relationships for describing particular microstructural processes have been developed and reported for conventional materials such as aluminum, copper, iron, nickel, and their dilute alloys, with steel receiving the most study.

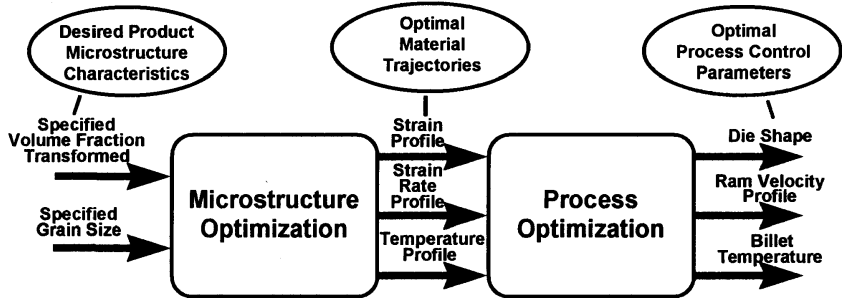

Fig. 1-Schematic representation of the two-stage approach

Within the specified ranges of temperature and strain rate, the operative deformation mechanisms of specialty alloys such as superalloys, intermetallics, ordered alloys, and metal matrix composites potentially become well defined and are amenable for modeling. ${ }^{[10,11]}$ As an illustration, consider a case of dynamic recrystallization for which a possible state-space model is

$$
\left[\begin{array}{c}
\dot{D} \\
\dot{\chi} \\
\dot{\varepsilon} \\
\dot{T}
\end{array}\right]=\left[\begin{array}{c}
f_{1}(T, \dot{\varepsilon}, D) \\
f_{2}(T, \dot{\varepsilon}, D, \chi) \\
u \\
\eta \sigma \dot{\varepsilon} / \rho C_{p}
\end{array}\right]
$$

The current state at any point in a deforming body is described by the state variables grain size $D$, volume fraction recrystallized $\chi$, accumulated strain $\varepsilon$, and current temperature $T$. The time rates of change of these variables, i.e., $\dot{D}, \dot{\chi}, \dot{\varepsilon}$, and $\dot{T}$, are functions of the current state variables and input variable, which is strain rate. In Eq. [1], $f_{1}$ and $f_{2}$ are known functions; $u$ is the system input, which is the strain rate in the present case; $\eta$ is a coefficient that determines how much of the mechanical work is converted into heat and contributes to the increase in temperature of the billet; $\sigma$ is flow stress; and the product $\rho C_{p}$ is the heat capacity ( $\rho$ is density and $C_{p}$ is the specific heat) of the material.

In addition to dynamic system models, the formulation of an optimal control problem requires a statement of physical constraints and specification of an optimality criterion for producing the desired hot-worked microstructural characteristics. The limiting process conditions for acceptable hot workability are important material behavior constraints in the first stage of the control strategy. Several methods for identifying ranges of temperature and strain rate over which the material exhibits a safe processing window have been reported. ${ }^{[10-14]}$ Any of these methods can be applied to identify the acceptable variation in processing parameters for safe processing, and then, a particular thermomechanical trajectory can be determined using the prescribed optimality criterion.

\section{B. Formulation of the Optimal Control Problem}

The design problem is formulated into an open-loop optimal control problem ${ }^{[1]}$ that can be formally stated as follows. Find input variables $u$ to minimize the optimality criterion

$$
J=h\left[x\left(t_{f}\right)\right]+\int_{t=0}^{t_{f}} g[x(t), u(t)] d t
$$




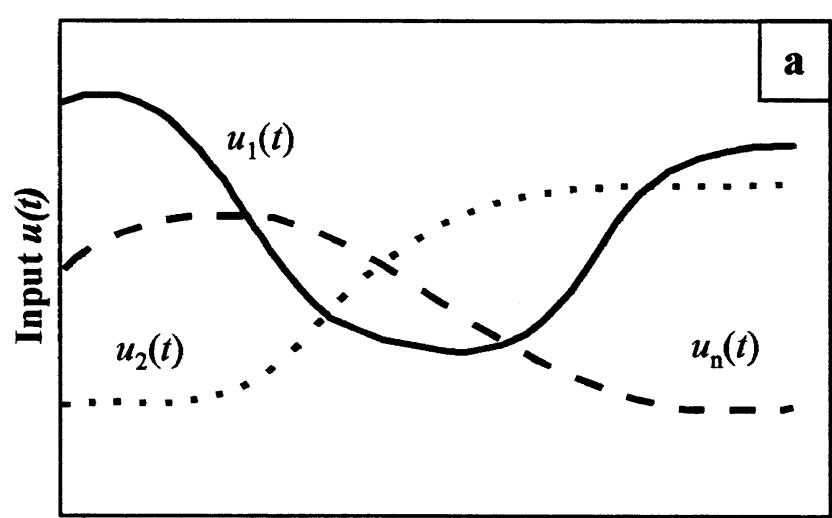

Time, $t$

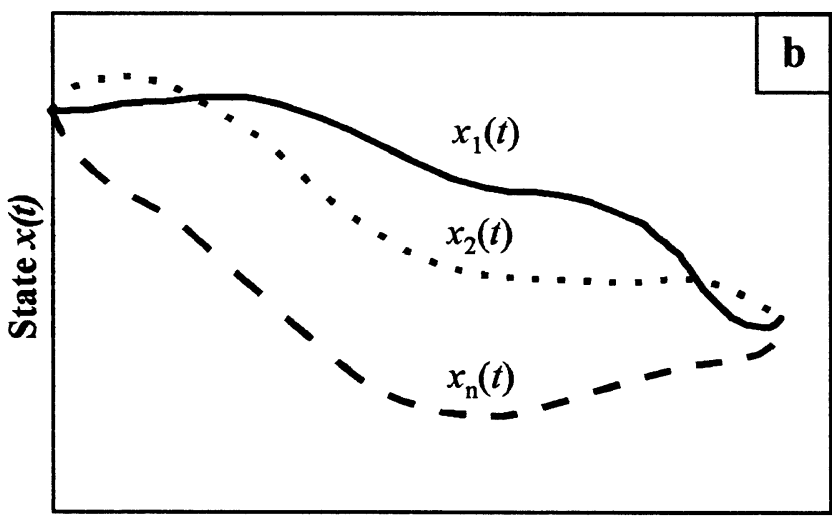

Time, $t$

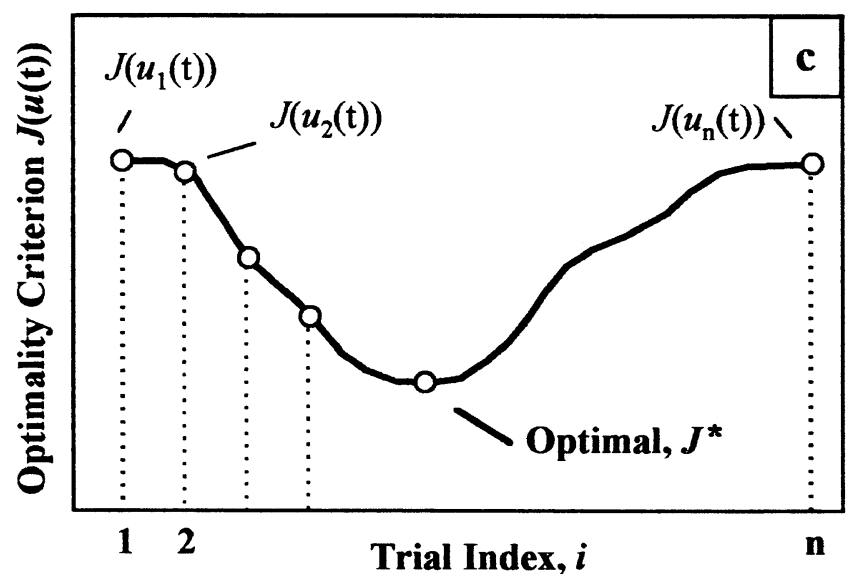

Fig. 2-A one-input, one-state example of the optimal control problem: (a) several possible input trajectories, (b) corresponding state trajectories, and $(c)$ corresponding values of the optimality criterion.

with the constraint that the system state obeys the statespace model:

$$
\dot{x}(t)=f[x(t), u(t)], x(0)=x_{0}
$$

In Eqs. [2] and [3], $t$ is time; $x(t)$ is a vector of state variables; $u$ is the system control input; $t_{f}$ is the duration of the process; $h\left(x\left(t_{f}\right)\right)$ is the penalty associated with violating the desired final state; $g(x(t), u(t))$ is the integrand of the penalty associated with deviating from desired state and control input trajectories; $f(x(t), u(t))$ is a vector function that describes the process dynamics; and $x_{0}$ is the initial state condition. A graphical description of the optimal con- trol problem represented by Eqs. [2] and [3] is given in Figure 2 for a case of a system with one input and one state. Suppose that an optimality criterion of the type given in Eq. [2] has been defined and that several possible input trajectories have been evaluated according to that optimality criterion. Figure 2(a) shows several of the infinite number of trajectories that the system input can follow. The corresponding trajectories of the state variable (called "trial trajectories") are given in Figure 2(b). Figure 2(c) gives the value of the optimality criterion that corresponds to each of these trial trajectories as a function of trial index, $i$. The objective is to find the input trajectory that, together with the corresponding trajectory of the state variable, will give a minimum value of $J$, labeled as "Optimal, $J^{*}$ ', in Figure 2(c). It is important to note that minimization of an optimality criterion implies that the system has been optimized with respect to that particular criterion, and that whether the original design objectives underlying the formulation of the optimality criterion have been met is a different issue.

\section{Optimality Criteria for Microstructure Development}

Careful selection of optimality criteria is critical for finding the most appropriate design solutions. In the control of microstructure development during hot metal deformation, common design criteria include producing specified microstructural features and gradients of microstructure within a specified variance on a repeatable basis. These optimality criteria and others can usually be formulated as functions to be minimized, and are often lumped together into a single scalar optimality criterion $J$ in the form

$$
J=J_{1}^{F}+J_{2}^{F}+\ldots+J_{N_{F}}^{F}+J_{1}^{T}+J_{2}^{T}+\ldots+J_{N_{T}}^{T}
$$

where the superscripts $F$ and $T$ refer to requirements on desired final states and trajectories, respectively. In the case where it is desired that microstructure feature $x$ achieve a value $x_{\text {des }}$ at the termination of the deformation process, the corresponding term in $J$ often has the form

$$
J_{i}^{F}=\beta_{i}\left(x\left(t_{f}\right)-x_{\mathrm{des}}\right)^{2}
$$

where $\beta_{i}$ is a weight factor. This type of function can also be used to include certain fixed process parameters and other final values for nonmicrostructural quantities such as strain and temperature in optimization calculations. The terms $J_{j}^{T}$ in the optimality criterion define requirements on the desired state and control input trajectories during the forming process and have integral forms.

Table I shows some examples of typical optimality criteria for microstructure development during hot metal deformation. Both final value and trajectory specifications are shown. The general formulation of this approach allows new terms to be defined according to the specific needs of each design problem. The quantities $f_{x}(x, a)$ and $f_{x}(x, a, b)$ in Table I are penalty functions that can be used to constrain optimized design solutions within acceptable process parameter ranges imposed by material workability or equipment limitations. These functions evaluate to virtually zero for values of $x$ in the acceptable range and attain very high values when $x$ is outside that range. Scalars $a$ and $b$ define the acceptable ranges for process parameters, such as temperature or strain rate. An example of a penalty function is shown in Figure 3. 
Table I. Examples of Typical Terms for the Optimality Criterion for Microstructure Development during Hot Metal Deformation

\begin{tabular}{lc}
\hline \multicolumn{1}{c}{ Design Objective } & Term in the Optimality Criterion \\
\hline Achieve final average grain size $D_{\text {des }}$ & $J_{i}^{F}=\beta_{i}\left(D\left(t_{f}\right)-D_{\text {des }}\right)^{2}$ \\
Achieve final strain of $\varepsilon_{\text {des }}$ & $J_{i}^{F}=\beta_{i}\left(\varepsilon\left(t_{f}\right)-\varepsilon_{\text {des }}\right)^{2}$ \\
Maintain strain rate between $\dot{\varepsilon}_{\text {min }}$ and $\dot{\varepsilon}_{\text {max }}$ because of workability considerations & $J_{j}^{T}=\int_{0}^{t_{f}} \beta_{j}(t) f\left(\dot{\varepsilon}, \dot{\varepsilon}_{\text {min }}, \dot{\varepsilon}_{\text {max }}\right) d t$ \\
Limit deformation heating; initial temperature is $T_{0}$ & $J_{j}^{T}=\int_{0}^{t_{f}} \beta_{j}(t)\left(T-T_{0}\right)^{2} d t$ \\
Keep strain rate under $\dot{\varepsilon}_{\text {max }}$ because of equipment limitations & $J_{j}^{T}=\int_{0}^{t_{f}} \beta_{j}(t) f\left(\dot{\varepsilon}, \dot{\varepsilon}_{\text {max }}\right) d t$ \\
Maintain temperature between $T_{\min }$ and $T_{\text {max }}$ because of workability considerations. & $J_{i}^{T}=\int_{0}^{t_{f}} \beta_{j}(t) f\left(T, T_{\text {min }}, T_{\text {max }}\right) d t$
\end{tabular}

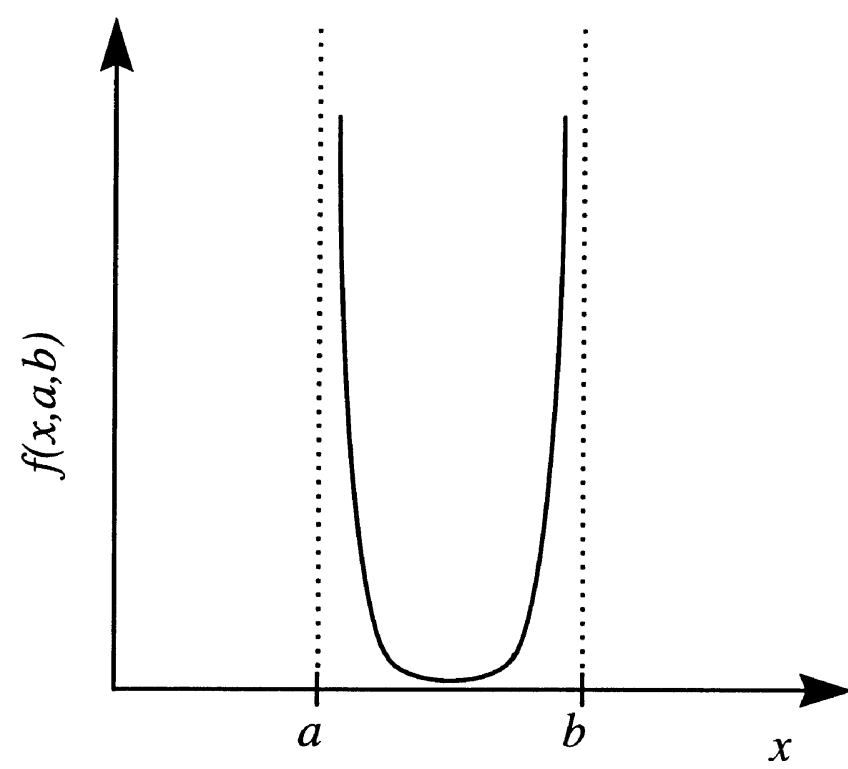

Fig. 3-A typical penalty function $(f(x, a, b))$ for constraining process parameters between $a$ and $b$.

The weight factors $\beta_{i}$ serve three purposes. First, they are used to scale the terms in $J$ so that they have comparable influence in the overall optimality criterion. Second, they are increased for certain terms according to their relative importance to achieve overall design requirements. Third, they may be adjusted in order to avoid possible conflicts in design requirements and obtain a satisfactory compromise solution.

The optimality criterion $J$, which is to be minimized in order to determine $\varepsilon$, $\dot{\varepsilon}$, and $T$, can incorporate a number of physically realistic requirements. For the specific problem of hot metal deformation, one possible optimality criterion is

$$
\begin{aligned}
J & =\beta_{1}\left(D\left(t_{f}\right)-D_{\mathrm{des}}\right)^{2}+\beta_{2}\left(\chi\left(t_{f}\right)-\chi_{\mathrm{des}}\right)^{2} \\
& +\int_{0}^{t_{f}}\left\{f_{2}^{w}\left[\dot{\varepsilon}(t), \dot{\varepsilon}_{\min }, \dot{\varepsilon}_{\mathrm{max}}\right]+f_{1}^{w}\left[T(t), T_{\min }, T_{\max }\right]\right\} d t
\end{aligned}
$$

In Eq. [6], $D$ is the average recrystallized grain size; $D_{\text {des }}$ is the desired final grain size; $\chi_{\text {des }}$ is the desired final volume fraction recrystallized; $\dot{\varepsilon}_{\text {min }}$ and $\dot{\varepsilon}_{\text {max }}$ are the minimum and maximum limits of strain rate, respectively; $T_{\min }$ and $T_{\max }$ are the minimum and maximum limits of temperature for acceptable workability, respectively; $\dot{\varepsilon}(t)$ is the nominal strain rate; and $T(t)$ is the nominal temperature. The functions $f_{1}$ and $f_{2}$ ensure that the nominal strain rate and temperature during deformation will be kept within the prescribed limits of the safe processing window.

Finding an analytical solution to the problem given in Eq. [6] is highly unlikely due to the complexity of the resulting functional form. However, it is possible to formulate a numerical algorithm that can yield a practical solution. The approach used here for obtaining a solution depends on two developments. First, a set of necessary conditions for optimality is obtained by applying variational principles, as given by Kirk; ${ }^{[1]}$ this formulation transforms the optimization problem to a problem of solving a set of constraint equations. Second, a numerical algorithm is developed for the solution of these equations. The solution methodology is briefly explained in the Appendix. A more detailed explanation is available elsewhere. ${ }^{[15]}$

\section{PROCESS DESIGN FOR HOT EXTRUSION OF STEEL}

The two-stage approach for microstructural control was applied to the hot extrusion of steel. An empirical model developed by $\mathrm{Yada}^{[16]}$ and critically reviewed and assessed by Devadas et al., ${ }^{[17]}$ shown in Table II, was used to de- 
Table II. Yada Equations for the Dynamic Recrystallization of Steel

\begin{tabular}{cc}
\hline $\begin{array}{c}\text { Volume fraction recrystallized } \\
\text { Critical strain }\end{array}$ & $\chi=1-\exp \left(\ln (2)\left(\left(\varepsilon-\varepsilon_{c}\right) / \varepsilon_{0.5}\right)^{2}\right)$ \\
$\varepsilon_{c}=4.76 \times 10^{-4} e^{8000 / T}$ \\
Plastic strain for 50 pct volume fraction recrystallization & $\varepsilon_{0.5}=1.144 \times 10^{-3} D_{0}^{0.28} \dot{\varepsilon}^{0.05} e^{6420 / T}$ \\
Average recrystallized grain size & $D=22,600 \dot{\varepsilon}^{-0.27} e^{-0.27(Q / \mathrm{R} T)}$ \\
Activation energy and gas constant & $Q=267 \mathrm{~kJ} / \mathrm{mol}, R=8.314 \times 10^{-3} \mathrm{~kJ} / \mathrm{mol} \mathrm{K}$ \\
\hline
\end{tabular}

scribe the relationship between microstructural parameters $D$ and $\chi$ and process parameters $\dot{\varepsilon}, T$, and $\varepsilon$. The volume fraction recrystallized $\chi$ is zero until a critical amount of strain $\varepsilon_{c}$ has been imposed. Beyond this critical strain, the kinetics of recrystallization at any temperature $T$ is characterized by $\varepsilon_{0.5}$, the strain required to reach 50 pct recrystallization. The time derivative of the fraction recrystallized can be approximated by applying the chain rule of differentiation to the equation for $\chi$ in Table II with some simplifying assumptions. The result is shown in Table III.

If the desired objective is to achieve a specified grain size, it is possible to obtain the desired $\dot{\varepsilon}$ from the fourth equation in Table II, assuming $T$ is fixed. Because of the dependence of $\dot{T}$ on $\dot{\varepsilon}$ (see Table III), this assumption is not valid, although it may be adequate in some applications. The purpose of this experiment, however, is to validate a systematic approach applicable to any problem, not to find a solution for plain carbon steel only. It should be clear that, for many materials, the dependence of the size of the forming grains on the temperature, strain rate, and strain may be considerably more complex. In fact, the coupling among these parameters combined with multiple constraints on temperature, strain rate, and strain can easily eliminate straightforward solution approaches, while the proposed approach can reliably solve the problem.

The equation for the rate of change of temperature due to deformation given in Table III states that a fraction $\eta$ of the mechanical work is converted to heat and increases the temperature. The expression for flow stress was obtained from Kumar et al. ${ }^{[18]}$ The microstructural state of the material is given by the state vector $\mathbf{x}=[\chi, T, \varepsilon]^{T}$, which evolves according to the equations given in Table III for time derivatives of $\chi$ and $T$ and the obvious relationship between the input strain rate $u$ and the evolution of strain. Since the grain size does not influence the other state variables $\chi, \varepsilon$, and $T$, it is treated as an output of the dynamical system and not included as one of the state variables.

\section{A. Stage 1: Optimizing the Microstructural Trajectories}

Since microstructure directly influences mechanical properties, the appropriate optimality criterion places a significant emphasis upon the final mechanical and microstructural states of the material. For the case studied here, the optimality criterion was chosen so as to attain a given final strain of 2, while assuring that the recrystallized grain size was kept at a desired value of $26 \mu \mathrm{m}$. The average grain size of the raw stock prior to extrusion was $120 \mu \mathrm{m}$. The optimality criterion chosen was

$$
J=10\left(\varepsilon\left(t_{f}\right)-2.0\right)^{2}+\int_{0}^{t_{f}}(D(t)-26)^{2} d t
$$

where a desired final strain of 2 , with a weighing factor of 10 , and a desired grain size of $26 \mu \mathrm{m}$ have been specified. The results of the first stage or microstructural optimization problem are shown in Figure 4(a). Starting at an initial temperature of $1273 \mathrm{~K}$, the temperature of the material increases approximately to $1295 \mathrm{~K}$ for deformation to a strain of 2.0. The strain rate is initially slightly below $1.0 \mathrm{~s}^{-1}$ and increases gradually to a little above $1.0 \mathrm{~s}^{-1}$. The recrystallized grain size, which is initially $120 \mu \mathrm{m}$, decreases to 26 $\mu \mathrm{m}$ beyond the critical strain of approximately 0.25 . Subsequently, since the recrystallized grain size depends both on $T$ and $\dot{\varepsilon}$, the simultaneous increase in both of these variables maintains the grain size constant. The results of two additional optimization runs to achieve grain sizes of 30 and $15 \mu \mathrm{m}$ are shown in Figures 4(b) and (c), respectively. The initial billet temperature was $1273 \mathrm{~K}$ for the second case and $1223 \mathrm{~K}$ for the third case.

\section{B. Stage 2: Optimizing the Process Parameters}

It is not physically possible to ensure that all the points in the deforming piece will undergo the strain, strain rate, and temperature trajectories obtained in stage 1. However, process parameters such as die geometry, ram velocity, and billet temperature can be designed to ensure that selected regions of the material will experience trajectories that approximate those designed. It is feasible to formulate a second optimization problem that determines values for process parameters that will attempt to achieve the desired trajectories at predetermined points in the material piece. In such an approach, each evaluation of the objective function for the optimization process usually requires a detailed analysis of the deformation process by the finite element method or some other technique.

In the case of round-to-round extrusion, it is possible to analytically calculate the die profile and ram velocity necessary for achieving the desired strain and strain rate profiles at the centerline of the piece. Given that $r_{0}$ is the die entrance radius (equal to the billet radius), $L$ is the die length, and $\varepsilon(t)$ is the required strain trajectory, the ram velocity is

$$
V_{\mathrm{ram}}=\frac{L}{\int_{t=0}^{t_{f}} e^{\varepsilon(t)} d t}
$$


Table III. Equations Used in the State-Space Model of Microstructural Evolution

Time derivative of volume fraction recrystallized

Time derivative of temperature

Flow stress $(\mathrm{kPa})$

$$
\dot{\chi} \approx \frac{\partial \chi}{\partial \varepsilon} \frac{d \varepsilon}{d t}=\frac{2 \ln 2}{\left(\varepsilon_{0.5}\right)^{2}}\left(\varepsilon-\varepsilon_{c}\right)(1-\chi) \dot{\varepsilon}
$$

$$
\dot{T}=\frac{\eta}{\rho C_{p}} \sigma(\varepsilon, \dot{\varepsilon}, T) \dot{\varepsilon}
$$

$$
\begin{gathered}
\sigma=\sinh ^{-1}\left[(\dot{\varepsilon} / A)^{1 / n} e^{Q / \mathrm{nR} T}\right] / 0.0115 \times 10^{-3} \\
\ln A(\varepsilon)=13.92+9.023 / \varepsilon^{0.502} \\
n(\varepsilon)=-0.97+3.787 / \varepsilon^{0.368}
\end{gathered}
$$

Activation energy and gas constant

$Q(\varepsilon)=125+133.3 / \varepsilon^{0.393}, R=8.314 \times 10^{-3}$
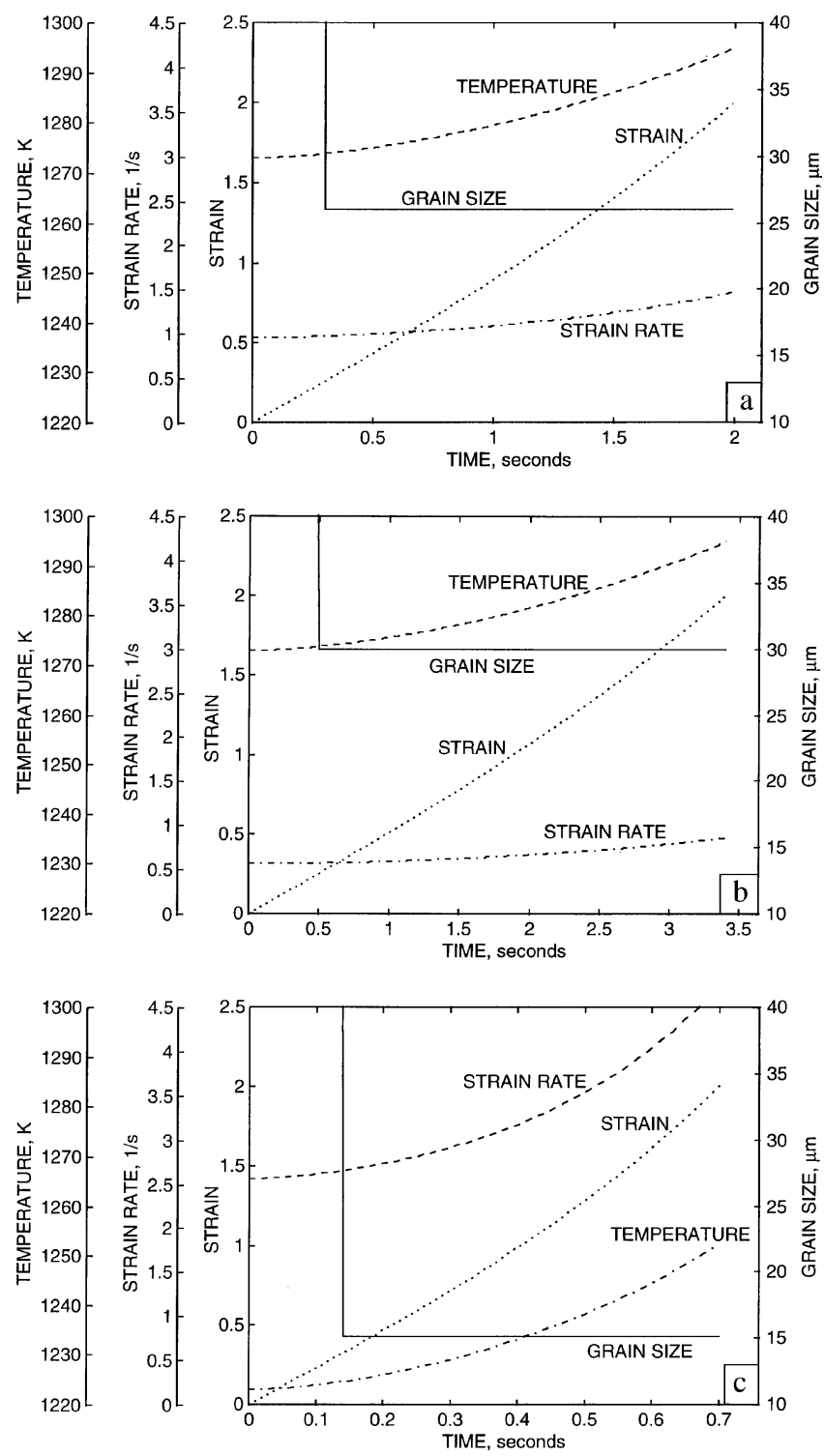

Fig. 4-Trajectories of strain, strain rate, temperature, and grain size for achieving the desired final grain size of (a) $26 \mu \mathrm{m},(b) 30 \mu \mathrm{m}$, and $(c)$ $15 \mu \mathrm{m}$.

The die shape can be described by the radius $r$ and axial distance (die throat length) $y$, where $r(t)=r_{0} e^{-\varepsilon(t) / 2}$ and $y(t)=V_{\mathrm{ram}} \int_{0}^{t} e^{\varepsilon(\tau)} d \tau$. Figure 5 gives the optimal die profile

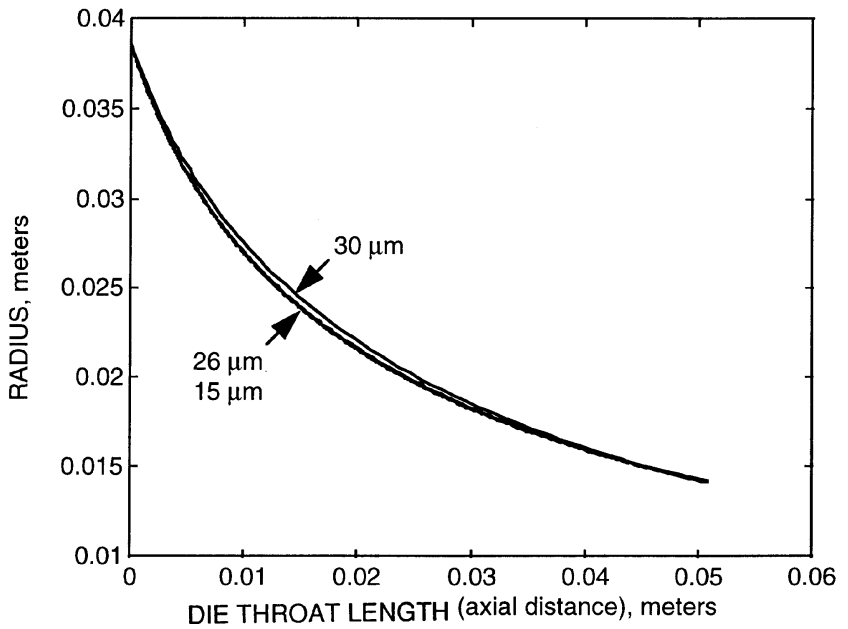

Fig. 5-Optimum die profile for achieving the final grain size of 26, 30, and $15 \mu \mathrm{m}$.

for achieving final grain sizes of 26,30 , and $15 \mu \mathrm{m}$, obtained using this approach. The optimum ram velocities for achieving these grain sizes were $8.43,5$, and $25.1 \mathrm{~mm} / \mathrm{s}$, respectively. Note that the die shape is almost the same for the three optimization cases. This means that the same die can be used to achieve the different recrystallized grain sizes by changing only the velocity of the extrusion press.

\section{EXPERIMENTAL VERIFICATION}

The optimization methodology was verified by means of an extrusion experiment. The extrusion test was performed on a $6000 \mathrm{kN}$ Lombard horizontal extrusion press located at Wright-Patterson Air Force Base, OH. The billet material was AISI 1030 steel (identical composition to the material used by Yada et al..$^{[16]}$ ), and the extrusion die was fabricated with $\mathrm{H} 13$ tool steel. The microstructure of the raw material is shown in Figure 6. The die geometry, generated to yield $26-\mu \mathrm{m}$ grain size, was utilized in this experiment with a ram velocity of $8.43 \mathrm{~mm} / \mathrm{s}$. The diameter and length of the billet were 74.15 and $150 \mathrm{~mm}$, respectively. The extrusion ratio was 7.6:1. The ambient temperature of the die, the container, and the follower block was $533 \mathrm{~K}$, and the soak temperature for the billet was $1273 \mathrm{~K}$. The extrusion was stopped at a ram stroke of $75 \mathrm{~mm}$ and resulted in the partial extrusion of the billet. The partial extrusion was carried out to study the microstructural development in the deformation zone (i.e., in the die region). The billet was subsequently 


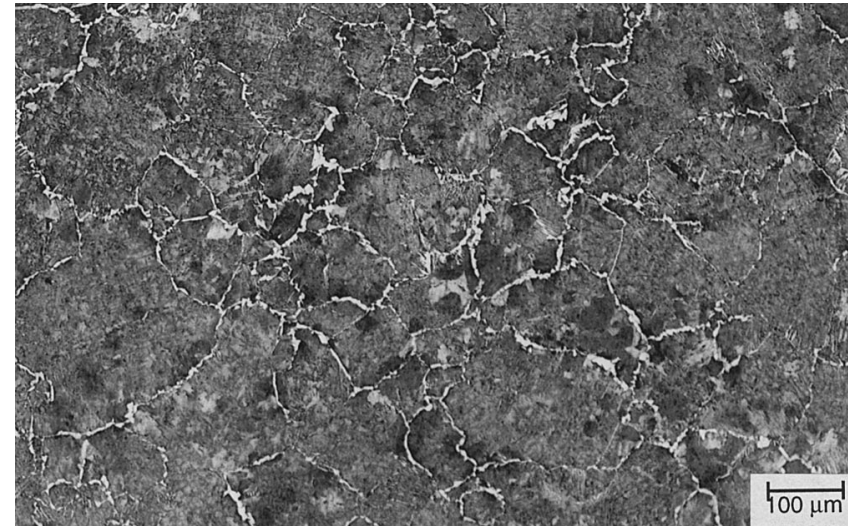

Fig. 6-Initial microstructure of the raw stock of 1030 steel.

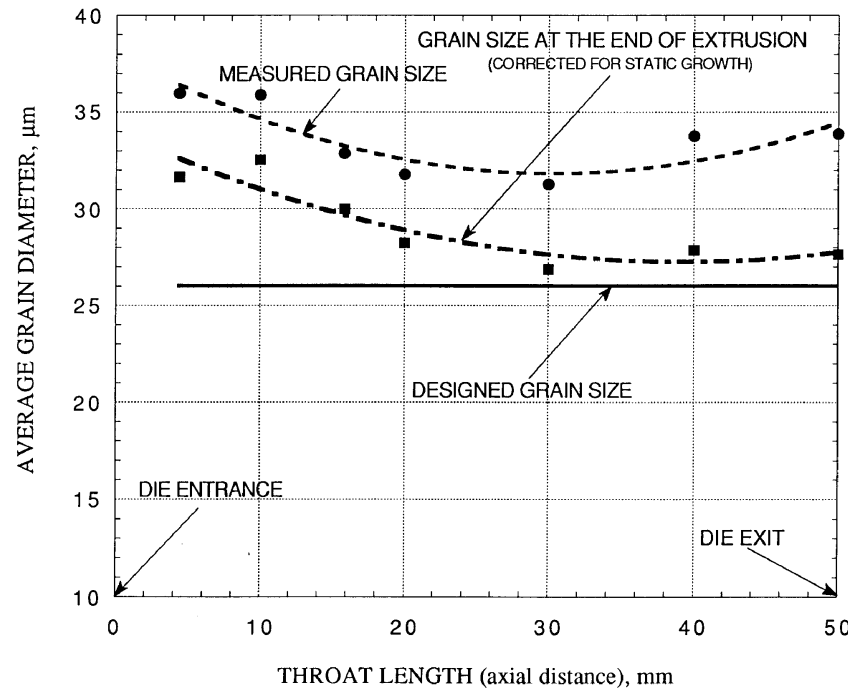

Fig. 7-Variation of measured and corrected grain size along the centerline of the partially extruded piece as a function of die throat length (axial distance).

removed from the container and was water quenched. The transfer time was 39 seconds. Another extrusion was allowed to proceed without interruption, and the extrudate was water quenched immediately after extrusion.

The partially extruded piece was cut along its longitudinal axis and polished for microstructural investigations. The polished sample was etched with 2 pct nital to reveal grain boundaries for grain size measurements. Using the Heyn intercept method, ${ }^{[19]}$ grain size measurements were carried out at various locations in the deformation zone of the partially extruded sample. The variation of grain size along the longitudinal axis, as a function of die throat length (axial position), was recorded as shown in Figure 7. Microstructural studies on the extruded sample revealed equiaxed grains and straight twin boundaries. A typical microstructure is given in Figure 8, corresponding to a location at the exit of the die. It is anticipated that the austenitic microstructure will experience static grain growth during the time interval between the stoppage of extrusion and quenching. The postdeformation effects can be corrected using Yada's model ${ }^{[16]}$ for static grain growth:

$$
D^{2}=D_{0}^{2}+A t e^{\frac{-Q_{g g}}{\mathrm{R} T}}
$$

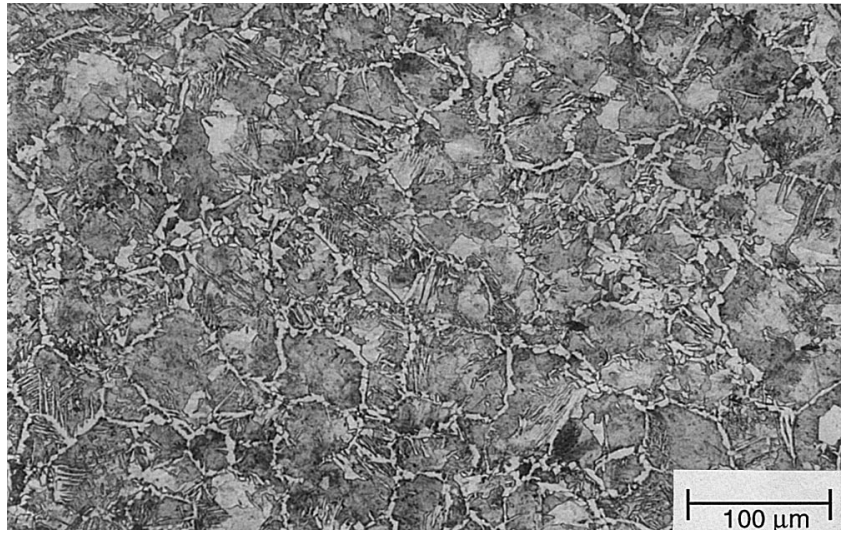

Fig. 8-Microstructure at a location corresponding to the exit of the die (end of deformation) of the partially extruded piece.

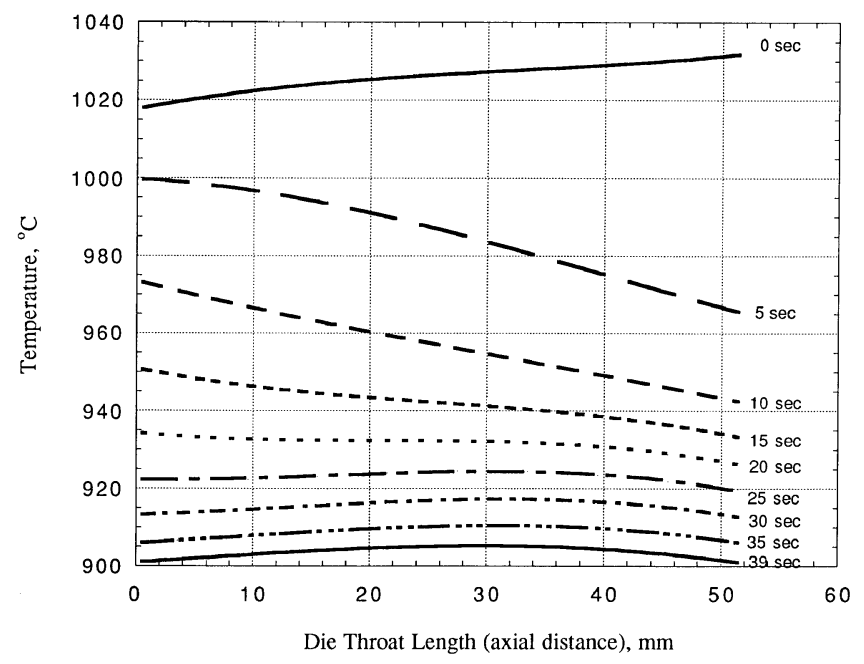

Fig. 9-Transient thermal history (predicted by finite element simulation) of the partially extruded billet during cooling after the completion of the deformation and prior to water quench.

where $D_{0}$ and $D$ are the recrystallized grain size upon extrusion and the statically grown grain size, respectively; $t$ is the time elapsed, in seconds, between the end of extrusion and the quenching operation; $A=1.44 \times 10^{12} \mu \mathrm{m}^{2} / \mathrm{s}$, and $Q_{g g} / R=32,100 \mathrm{~K}$.

The partial extrusion was simulated using the "Antares" process simulation software ${ }^{[20]}$ for the nonlinear coupled response of the billet and the thermal response of the die. After partial extrusion, the temperature at the billet centerline increased to $1313 \mathrm{~K}$ due to deformation heating. The continuous cooling of the partially extruded billet for a period of 39 seconds prior to water quench was simulated. Figure 9 shows the resultant transient thermal behavior at the billet centerline during cooling. These temperature data were utilized for correcting the increase in grain size due to static grain growth. The variation of the estimated grain size at the completion of the deformation (i.e., the grain size of the extrudate if it had been quenched immediately after the deformation) as a function of die throat length (axial distance) is also given in Figure 7. The average grain size of $27.5 \mu \mathrm{m}$ is in good agreement with the $26-\mu \mathrm{m}$ grain size for which the extrusion process was designed.

Microstructural examination was also carried out along the centerline of the extruded rod of approximately $2 \mathrm{~m}$ in 

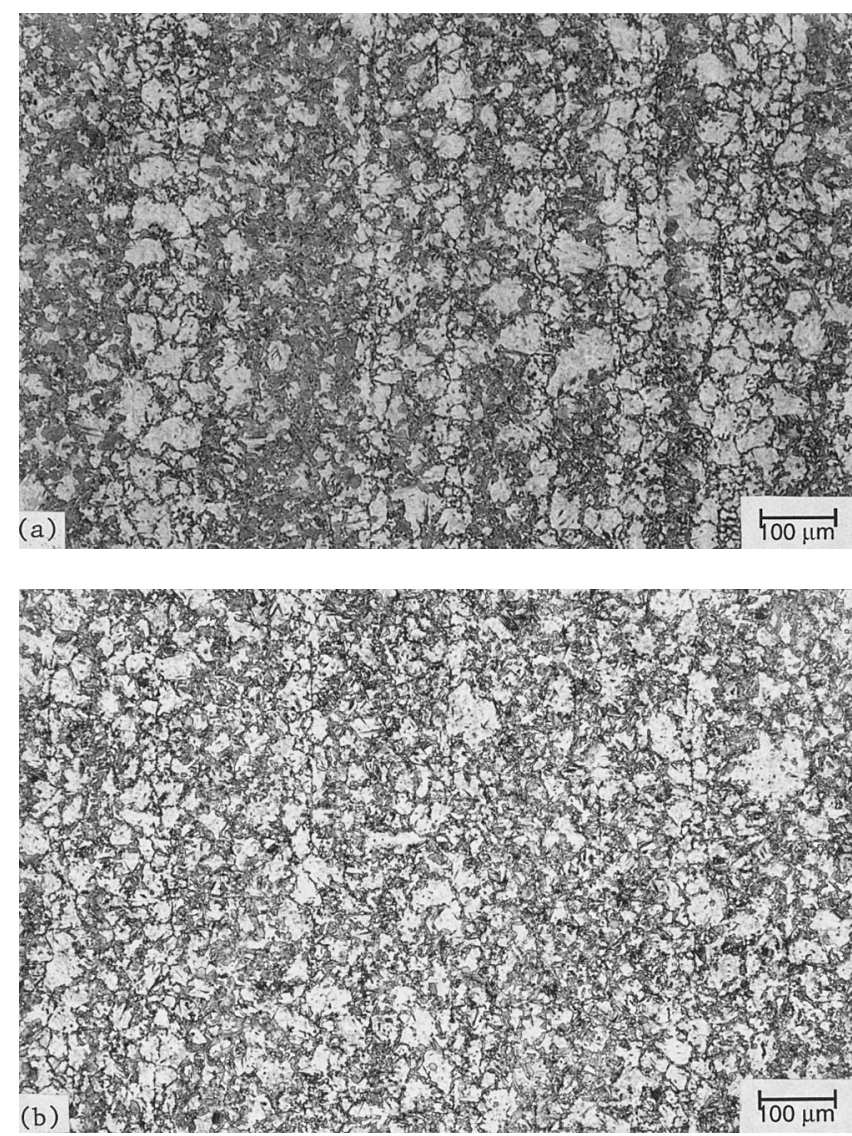

Fig. 10 - Typical microstructures of the products extruded at a temperature and ram velocity of $(a) 1273 \mathrm{~K}$ and $8.3 \mathrm{~mm} / \mathrm{s}$ and $(b) 1223 \mathrm{~K}$ and 25.1 $\mathrm{mm} / \mathrm{s}$.

length obtained from the uninterrupted test. There is no variation in microstructure along the length of the extrudate. A typical microstructure of the extruded rod is given in Figure 10(a). The measured grain size was $27 \mu \mathrm{m}$, which is close to the designed value.

The die profile (designed for yielding a grain size of 26 $\mu \mathrm{m})$ can be used to obtain the final grain size of $15 \mu \mathrm{m}$ (refer to Figure 5) in the extrudate if the billet temperature is $1223 \mathrm{~K}$ and the ram velocity is $25.1 \mathrm{~mm} / \mathrm{s}$. Another extrusion was also carried out under the same conditions. The extruded rod was water quenched immediately after extrusion. Microstructural examination of the extrudate revealed that the microstructure is uniform throughout the rod of approximately $2 \mathrm{~m}$ in length. A typical microstructure is given in Figure 10(b). The measured average grain size was $17 \mu \mathrm{m}$.

\section{SUMMARY}

A two-stage approach based on optimal control theory has been proposed in order to control microstructure development during hot working. This method was utilized for optimal design of a hot extrusion process. In the first stage, Yada's equations for dynamic recrystallization of plain carbon steel were utilized to obtain an optimal deformation path to achieve a grain size of $26 \mu \mathrm{m}$ in the product. This trajectory determination was performed via minimization of an optimality criterion. In the second stage, a geometric mapping was utilized to develop an extrusion die profile such that the strain rate profile during extrusion matches the optimal trajectory computed in the first stage.

Validation experiments were performed by utilizing the extrusion die geometry obtained in the second stage. The measured grain sizes of the extruded products were observed to be in close agreement with the designed values. Therefore, it can be concluded that the proposed two-stage approach using the principles of control theory can be reliably applied to optimize and control microstructure during extrusion. Extension to other metal forming processes will require the integration of advanced analytical or simulation methods into the second stage of the design methodology. It is also anticipated that the current approach can be extended to incorporate postdeformation effects.

\section{ACKNOWLEDGMENTS}

Support for this work was provided by the Materials Process Design Branch, Materials Directorate, Wright Laboratory (Wright-Patterson Air Force Base, OH). Universal Energy Systems, Inc. and Austral Engineering and Software, Inc. participated in this effort as part of a Small Business Innovative Research Program. One of the authors (SV) is thankful to the National Research Council for its support.

\section{APPENDIX}

\section{A. Solution of the Microstructure Development Trajectory Optimization Problem}

The definition of the optimal control problem is given by Eqs. [2] and [3] in the main text of this article. The approach used in this work for the solution of the optimal control problem follows that of Kirk. ${ }^{[1]}$ First, the original constrained minimization problem is transformed into an equivalent unconstrained minimization problem by appending the microstructural evolution equations via Lagrange multipliers to the design specifications to form a modified cost functional. A set of conditions necessary for optimality is then obtained; this amounts to transforming the unconstrained optimization problem into a problem that consists of finding the solution to a boundary value problem. Finally, the set of constraint equations is solved by using a numerical algorithm. Details of these developments are explained as follows.

In order to transform the problem of minimizing Eq. [2] under the constraints of Eq. [3] into a purely integral form, first assume that $h$ is a differentiable function. Next, introduce the Lagrange multipliers $p_{1}(t), p_{2}(t), \ldots, p_{n}(t)$, which are referred to as costates. It can be shown that minimizing $J$ is equivalent to minimizing the augmented functional

$$
\begin{aligned}
J_{a}(u)=\int_{t_{0}}^{t_{f}} & \left\{g(x(t), u(t), t)+\left[\frac{\partial h}{\partial x}(x(t), t)\right]{ }^{T} \dot{x}(t)\right. \\
+ & \left.\frac{\partial h}{\partial t}(x(t), t)+p^{T}(t)[f(x(t), u(t), t)-\dot{x}(t)]\right\} d t
\end{aligned}
$$

where $t_{0}$ and $t_{f}$ are the initial and final times, respectively. 
For convenience, the Hamiltonian function,

$$
H(x(t), u(t), p(t), t) \equiv g(x(t), u(t), t)+p^{T}(t) f(x(t), u(t), t)
$$

is often introduced in these problems. It is possible to show that, in order for $u(t)$ to minimize $J_{a}(u)$, and consequently $J(u)$, it is necessary that

$$
\begin{gathered}
\dot{x}(t)=\frac{\partial H}{\partial p}(x(t), u(t), p(t), t) \\
\dot{p}(t)=-\frac{\partial H}{\partial x}(x(t), u(t), p(t), t)
\end{gathered}
$$

$$
\begin{aligned}
& \frac{\partial H}{\partial u}(x(t), u(t), p(t), t) \\
& \quad=\frac{\partial g}{\partial u}(x(t), u(t), t)+p(t) \frac{\partial f}{\partial u}(x(t), u(t), t)=0
\end{aligned}
$$

for all $t \in\left[t_{0}, t_{f}\right]$, it is necessary that

$$
p\left(t_{f}\right)=\frac{\partial h}{\partial x}\left(x\left(t_{f}\right), t_{f}\right)
$$

and that

$$
x\left(t_{0}\right)=x_{0}
$$

Conditions [A3] through [A5] apply in general; conditions [A6] and [A7] are necessary when the final states are free and the final time is fixed, as is the case in this application.

Since these conditions are only necessary, any strain-rate trajectory $u(t)$ that solves the problem under consideration will satisfy conditions [A3] through [A7]. However, satisfaction of these conditions alone does not guarantee that an optimal trajectory has been found. In addition, it is important to recognize that these are only conditions that must be satisfied, and not a method for obtaining an optimal solution.

\section{B. Solution of the Microstructural Trajectory Optimization Problem}

Finding an analytical solution to the problem posed in Section A is highly unlikely due to the complexity of the resulting functional forms. However, it is possible to formulate a numerical algorithm that can yield a practical solution to the problem. The approach used here is based upon the idea of satisfying all of the conditions but one, and then bringing the remaining condition closer to satisfaction at each iteration. This type of algorithm is based on the notion of the first variation of a functional; the basic idea behind the algorithm can be stated as follows.

Given an initial guess $u^{(0)}$ for the optimal control trajectory, calculate a change in $u, \Delta u^{(0)}$, such that $u^{(0)}+\Delta u^{(0)}$ decreases the value of $J_{a}(u)$, i.e., $J_{a}\left(u^{(0)}\right.$ $\left.+\Delta u^{(0)}\right)<J_{a}\left(u^{(0)}\right)$. Update $u$ by $u^{(1)}=u^{(0)}+\Delta u^{(0)}$. Repeat this process until no further decrease in $J_{a}$ is obtainable.

It can be shown that, if conditions [A3], [A4], and [A6] are satisfied, which is not difficult to ensure, then the variation of $J_{a}$ is

$$
\delta J_{a}=\int_{0}^{t_{f}} \frac{\partial H}{\partial u} \delta u d t
$$

and one obvious choice of $\delta u$ that will decrease $J_{a}$ is

$$
\delta u=-\frac{\partial H}{\partial u}
$$

One way to think about this $\delta u$ is that it is the change in the shape of $u$ that decreases $J_{a}$ the most rapidly. Because this is only a first-order variation, the range over which it is accurate is limited, and it becomes necessary to select a step length $\tau$ that limits the size of the change in the shape of $u$ in the direction given by $\delta u$ to ensure that

$$
J_{a}(u+\Delta u)<J_{a}(u)
$$

where

$$
\Delta u=\tau \delta u
$$

A flowchart that describes a general step-length-based descent algorithm is shown in Figure A1. In the case where Eq. [A9] is used as the direction in which the control trajectory is modified, the algorithm is known as the steepest descent method. It can be shown that the steepest descent method converges globally at a linear convergence rate. Because faster convergence is usually desired, other methods are often used; these methods are discussed in optimization literature.

As mentioned previously, a step length $\tau$ has to be de-

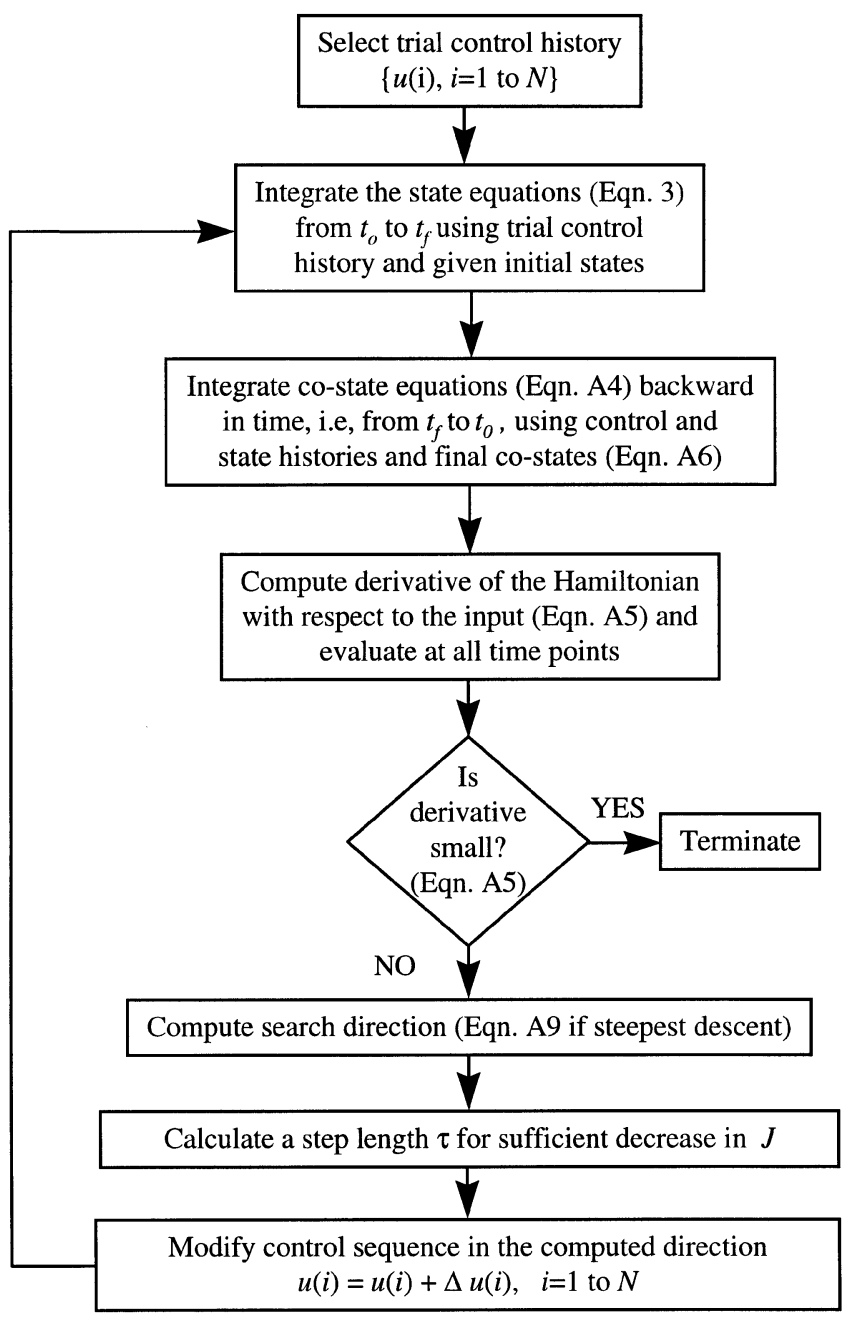

Fig. A1-Flowchart for general step-length-based descent algorithm. 
termined before the input trajectory can be modified in the computed search direction. This is usually accomplished by performing a unidimensional search in the prescribed direction until a value of $\tau$ is found that yields the greatest reduction in the objective function; a proven search method and convergence criteria are necessary for this univariate search procedure to be effective.

\section{REFERENCES}

1. D.E. Kirk: Optimal Control Theory: An Introduction, Prentice-Hall Inc., Englewood Cliffs, NJ, 1970, pp. 29-46 and pp. 184-309.

2. P.M. DeRusso, R.J. Roy, and C.M. Close: State Variables for Engineers, John Wiley and Sons, New York, NY, 1965.

3. W.G. Frazier, E.A. Medina, J.C. Malas, and R.D. Irwin: JMEP, 1997 , vol. 6 (2), pp. 153-60.

4. R.D. Irwin, R.D. Glenn, W.G. Frazier, D.A. Lawrence, and R.F. Follett: AIAA J. Guidance, Control, Dynamics, 1995, vol. 18 (2), pp. $214-21$

5. J.J. Jonas, C.M. Sellars, and McG. Tegart: Metall. Rev., 1969, vol. 14 (1), 1969, pp. 1-24

6. P.L. Orsetti Rossi and C.M. Sellars: Acta Mater., 1997, vol. 45 (1), pp. 137-48.

7. H.J. McQueen and J.J. Jonas: Treatise on Materials Science and Technology, Vol. 6, Plastic Deformation of Materials, Academic Press, New York, NY, 1975, pp. 393-493.
8. W. Roberts: in Deformation, Processing and Structure, G. Krauss, ed., ASM INTERNATIONAL, Metals Park, OH, 1984, pp. 109-84.

9. W. Roberts: Process Control in Steel Industry, MEFOS, Luleå, Sweden, 1986, vol. 2, pp. 551-77.

10. J.C. Malas: Ph.D. Dissertation, Ohio University Athens, OH, 1991.

11. J.C. Malas and V. Seetharaman: JOM, 1992, vol. 44 (6), pp. 8-13.

12. H.J. Frost and M.F. Ashby: Deformation Mechanism Maps; The Plasticity and Creep of Metals and Ceramics, Pergamon Press, Oxford, United Kingdom, 1982.

13. R. Raj: Metall. Trans. A, 1981, vol. 12A, pp. 1089-97.

14. Y.V.R.K. Prasad, H.L. Gegel, S.M. Doraivelu, J.C. Malas, J.T. Morgan, K.A. Lark, and D.R. Barker: Metall. Trans. A, 1984, vol. $15 \mathrm{~A}$, pp. $1883-92$.

15. W.G. Frazier: Robust Control Techniques for Hot Deformation Processes, Contributive Research and Development, vol. 228, Systran Corp., Dayton, OH, Final Report, Task 178, Contract No. F3361590-C5944, Mar. 1995.

16. H. Yada: Proc. Int. Symp. Accelerated Cooling of Rolled Steels, Conf. of Metallurgists, CIM, Winnipeg, MB, Canada, Aug. 24-26, 1987, G.E. Ruddle and A.F. Crawley, eds., Pergamon Press, Toronto, 1987, pp. $105-20$

17. C. Devadas, I.V. Samarasekara, and E.B. Hawbolt: Metall. Trans. A, 1991, vol. 22A, pp. 335-49.

18. A. Kumar, K.P. Rao, E.B. Hawbolt, and I.V. Samarasekara: Univ. of British Columbia, Vancouver, B.C., unpublished research, 1987.

19. George F. Vander Voort: in Metallography, Principles and Practice, McGraw-Hill Book Co., New York, NY, 1994, p. 410.

20. Antares Software User Manual, UES, Inc., Dayton, OH, 1995. 